\title{
Potential magnetic field calculator for solar physics applications using staggered grids
}

\author{
Callum M. Boocock and David Tsiklauri
}

\begin{abstract}
School of Physics and Astronomy, Queen Mary University of London, G.O. Jones Building, 327 Mile End Road, London E1 4NS, UK

e-mail: c.boocock@qmul.ac.uk
\end{abstract}

Received 19 November 2018 / Accepted 18 March 2019

\begin{abstract}
A program has been designed to generate accurately a potential magnetic field on a staggered grid by extrapolating the magnetic field normal to the photospheric surface. The code first calculates a magnetic potential using the Green's function method and then uses a finite differencing scheme to calculate the magnetic field from the potential. A new finite differencing formula was derived which accounts for grid staggering; it is shown that this formula gives a numerical approximation that is closest to the real potential field. It is also shown that extending the region over which normal photospheric field is specified can improve the accuracy of the potential field produced. The program is a FORTRAN 90 code that can be used to generate potential magnetic field inputs for Lare $3 \mathrm{~d}$ and other MHD solvers that use a staggered grid for magnetic field components. The program can be parallelised to run quickly over multiple computing cores. The code and supporting description are provided in the appendices.
\end{abstract}

Key words. Sun: magnetic fields - methods: numerical - magnetohydrodynamics (MHD)

\section{Introduction}

The coronal heating problem, the question of why the Sun's corona is much hotter $(\sim 1 \mathrm{MK})$ than photosphere $(\sim 6000 \mathrm{~K})$, is an ongoing problem in solar physics. Researchers agree that energy is transported to the corona by non-thermal transport of energy through the Sun's magnetic field (Arregui 2015), although the dominant mechanism for coronal heating is under debate (Parnell \& De Moortel 2012). Computational 3D magnetohydrodynamic (MHD) models are often used to address the coronal heating problem (Klimchuk 2015).

Computational MHD models require an initial magnetic field to be specified. For many problems, primarily those concerning MHD waves, it is useful to have a static equilibrium on which to impose perturbations to the velocity and magnetic fields (Goossens 2003). In the case of a static equilibrium both the velocity vector $\mathbf{v}$ and its time derivative $\partial \mathbf{v} / \partial t$ must equal zero.

Analytic expressions exist for a number of structures that describe magnetostatic equilibria in both 2D and 3D for example (Smith et al. 2007; Petrukhin et al. 2018; Oliver et al. 1998; Cuperman et al. 1989; Gent et al. 2013). For more detailed magnetic structures we need information from solar observations. Maps of the line-of-sight and vector magnetograms from the photosphere can be measured by means of spectropolarimetric methods such as the Zeeman effect, i.e. the splitting of spectral lines in the presence of a magnetic field (Beckers 1971). It is however much more difficult to measure directly the magnetic field in the solar corona (Ruan et al. 2008). Fortunately the photospheric magnetic field can be used to reconstruct the coronal field by means of extrapolation. Extrapolation of the photospheric magnetic field is currently the primary tool for modelling the coronal magnetic fields (Tadesse et al. 2014).

There are many methods for extrapolating the structure of the magnetic field from surface measurements. The commonly used methods rely on various assumptions (Neukirch 2005).
Most commonly non-magnetic forces such as pressure gradients and gravity are neglected; this is well justified in the solar corona because of the low plasma beta (Wiegelmann 2008) and when considering scales smaller than the hydrostatic scale height (Peter et al. 2015). For magnetostatic equilibrium the magnetic Lorentz force must then be zero $\mathbf{J} \times \mathbf{B}=0$. This defines a force-free field (Wiegelmann \& Sakurai 2012). Another way of expressing this condition is given below in Eq. (1) where $\alpha$ can be either zero, a constant, or a variable that is constant along field lines. These cases correspond to potential, linear, and non-linear force-free fields, respectively (Aschwanden 2004). This expression is written as

$\nabla \times \mathbf{B}=\alpha \mathbf{B}$.

Various methods of extrapolation are used to reconstruct potential fields, linear force-free fields (Gary 1989), and nonlinear force-free fields (Wiegelmann 2008). There are also methods of extrapolation available to reconstruct non-force-free fields although these may require additional data (Wiegelmann 2004). Furthermore several of these methods can be extended from Cartesian to spherical coordinates so that the solar coronal field can be calculated globally (Wiegelmann 2007; Wiegelmann et al. 2007).

In the particular case of a potential force-free field there is zero current $\mathbf{J}=\nabla \times \mathbf{B}=0$. The field is called a potential magnetic field because the magnetic field can be expressed in terms of a scalar potential $\mathbf{B}=\nabla \phi$. Potential magnetic fields are useful because they can be used, for example as an initial topology for MHD simulations (Gudiksen \& Nordlund 2005; Masson et al. 2009; Bingert \& Peter 2011, 2013; Bourdin et al. 2013), to study MHD wave phenomena (Thackray \& Jain 2017; Ofman \& Selwa 2008; Smith et al. 2007; Petrukhin et al. 2018), to embed non-potential fields (Browning et al. 2008), or to generate non-force-free MHD equilibria (Gordovskyy et al. 2014; 
Solanki \& Steiner 1990; Khomenko \& Collados 2006; Pizzo 1986; Wiegelmann \& Neukirch 2006; Inoue 2016; Inoue et al. 2013).

To calculate a potential magnetic field from the normal magnetic field $B_{n}$ at the photosphere many methods can be utilised such as the Fourier expansion, spherical harmonic expansions, and Green's function. The Fourier method and the Green's function method are compared in Sakurai (1982). Extrapolated fields require that the boundary conditions for extrapolation are able to match those of the MHD simulation (Otto et al. 2007).

In this work we consider the Green's function method, which uses a discrete approximation for $B_{n}$ within each mesh. This method is better suited for considering non-periodic, isolated magnetic regions but has the drawback of assuming that $B_{n}=0$ outside the region of interest. This method was first used by Schmidt (1964), was later developed for oblique photospheric data by Semel (1967), and adapted further by Sakurai who adjusted the method for practical applications on a finite numerical grid (Sakurai 1982).

This paper describes a potential field calculator based on a modified Green's function method. The purpose of this paper is to demonstrate an improved method of finite differencing suited to a MHD solver using a staggered magnetic field. It also demonstrates that by increasing the extent of input data relative to the computational domain the errors associated with assuming $B_{n}=0$ outside the region of interest can be reduced.

The different methods of numerical differentiation that were considered for the potential field calculator are explained and compared with the most accurate methods being chosen for the final implementation. The calculator has been designed to produce initial magnetic field inputs for Lare3d (Arber et al. 2001) but works with any MHD solver that uses staggered grids for the magnetic field components.

\section{Green's function method}

Taking $\mathbf{J}=\nabla \times \mathbf{B}=0$ as an initial condition, the Gauss law of magnetism can be expressed as the Laplace equation for the magnetic potential (Aschwanden 2004).

$\nabla \cdot \mathbf{B}=\nabla^{2} \phi=0$

By solving the Laplace equation for the scalar potential $\phi$, a potential magnetic field $\mathbf{B}$ can be calculated that results in a static equilibrium. Consider a 3D domain with $z$ vertical and the photospheric boundary at $z=0$. We denote the magnetic field normal to the photospheric boundary as $B_{z}(x, y, z)$, and the normal field at the photospheric boundary is then $B_{z 0}(x, y)=$ $B_{z}(x, y, 0)$.

The Laplace equation with the photospheric boundary condition $B_{z 0}=\partial \phi / \partial z$ can be solved numerically on a discrete grid of points $(i, j, k)$ with grid separation $d$ by using the modified Green's function method given below in Eq. (3) (Sakurai 1982), i.e.

$\phi(i, j, k)=\sum_{i_{1}} \sum_{j_{1}} \frac{B_{z 0}\left(i_{1}, j_{1}\right) d}{2 \pi \sqrt{\left(i-i_{1}\right)^{2}+\left(j-j_{1}\right)^{2}+\left(k+\frac{1}{\sqrt{2 \pi}}\right)^{2}}}$,

where $i_{1}$ and $j_{1}$ are dummy variables used to sum over contributions from each point at the photosphere. The normal magnetic field contributes to the potential via a discrete approximation of the Green's function. By virtue of the chosen Green's function the potential satisfies the Laplace equation and boundary conditions at $z=0$ and at $r \rightarrow \infty$ as follows:

$$
\begin{aligned}
-\mathbf{n} \cdot \nabla \phi & =B_{z 0} \quad(z=0) \\
\lim _{r \rightarrow \infty} \phi(\mathbf{r}) & =0 \quad(z>0)
\end{aligned}
$$

This particular choice of Green's function is representative of a "submerged magnetic source" at a depth $d / \sqrt{2 \pi}$ beneath each grid point at $z=0$. There is a slight inconvenience with this choice of Green's function in that the calculated normal magnetic field does not exactly match the original normal field at $z=0$. Whilst there are other possible choices that give a closer match, one of which is described in Sakurai (1982), this particular Green's function has been chosen for computational convenience because the focus of this paper is not on the choice of Green's function but rather the method of finite differencing used to differentiate the magnetic potential.

Once the magnetic potential is determined at each grid point the magnetic field components can then be calculated as derivatives of the magnetic potential,

$B_{x}=\frac{\partial \phi}{\partial x}, \quad B_{y}=\frac{\partial \phi}{\partial y}, \quad B_{z}=\frac{\partial \phi}{\partial z}$.

If a finite differencing scheme is used to differentiate the magnetic potential then the potential needs to be calculated in additional "ghost" cells at the boundaries of the domain. The number of additional cells depends upon the order of the differencing scheme. Ghost cells are also required in the input for Lare3d as described in Sect. 3.

As the potential must be calculated in the ghost cells, the potential due to the magnetic source, represented by the Green's function, must also be positioned beneath these cells and outside of the domain. This can be done by either increasing the indices in the $z$ direction so that the ghost cells are raised above the magnetic source or by altering Eq. (3) to effectively lower the magnetic source by the required number of cells.

In either case the overall effect is to consider the lowest ghost cell as the new position of the initial normal field. The magnetic field considered in the MHD solver is then be shifted up from the photosphere by the length of the additional ghost cells. Whilst this inaccuracy in position is inconvenient and there are ways to avoid this, for example manually fixing boundary conditions in Lare3d or not differentiating to determine the magnetic field in the lowest two layers, we decided to make this modification on the basis that any manual fixing of the boundary conditions affects the potential field equilibrium in ways that are independent of the methods of the finite differencing under investigation. The modified Green's function method used in this paper is therefore a slight modification of Eq. (3), which submerges the magnetic source represented by the Green's function by an additional $n$ cell depths, where $n$ is the maximum number of ghost cells required, i.e.

$\phi(i, j, k)=\sum_{i_{1}} \sum_{j_{1}} \frac{B_{z 0}\left(i_{1}, j_{1}\right) d}{2 \pi \sqrt{\left(i-i_{1}\right)^{2}+\left(j-j_{1}\right)^{2}+\left(k+n+\frac{1}{\sqrt{2 \pi}}\right)^{2}}}$.

\section{Staggered grids}

The computational domain in Lare $3 \mathrm{~d}$ is of size $(n x, n y, n z)$ however the magnetic field is defined on a staggered grid so that the magnetic field components are collocated at the face-centres of each cell. For example, the values of $B_{x}$ are shifted away from the cell centre by half a cell in the $x$ direction, as shown in Fig. 1, and similarly for $B_{y}$ and $B_{z}$ in the $y$ and $z$ directions, respectively. The magnetic field components are staggered in this way to maintain the solenoidal property of the magnetic field $\nabla \cdot \mathbf{B}=0$ 


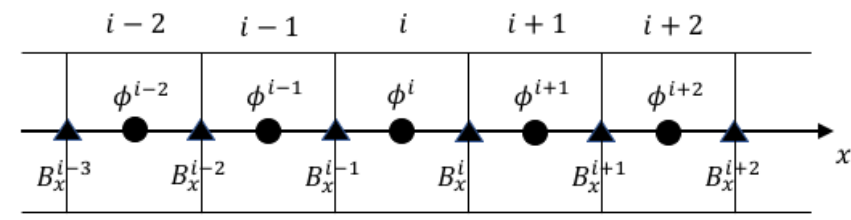

Fig. 1. One-dimensional representation of a small section around the $i$ th cell in the $x$ direction showing the position of $\phi$ values at the cell centres and $B_{x}$ values at the cell boundaries in the $x$ direction.

as it is evolved via the induction equation (Balsara \& Kim 2004). The grid staggering changes the position at which the magnetic field components must be specified in relation to the magnetic potential. Owing to the staggered grid the extents of $B_{x}, B_{y}$ and $B_{z}$ within the computational domain are written as

$B_{x}(0: n x, 1: n y, 1: n z)$,

$B_{y}(1: n x, 0: n y, 1: n z)$,

$B_{z}(1: n x, 1: n y, 0: n z)$.

The numerical grid on which the initial magnetic field must be specified in Lare $3 \mathrm{~d}$ is however larger. This is because Lare3d is second order accurate in space and therefore requires two ghost cells at each boundary. The extents of $B_{x}, B_{y}$ and $B_{z}$ to be specified in the initial magnetic field are therefore written as

$B_{x}(-2: n x+2,-1: n y+2,-1: n z+2)$,

$B_{y}(-1: n x+2,-2: n y+2,-1: n z+2)$,

$B_{z}(-1: n x+2,-1: n y+2,-2: n z+2)$.

\section{Comparison methodology}

In order to compare the effects of different methods of numerical differentiation and the extent of the region over which the normal photospheric field is specified, we need to produce and compare different potential fields. Our aim is to maximise the accuracy of the potential field calculator. A potential magnetic field should be current free, should not produce motion in a static plasma, and should not evolve with time.

\subsection{Test cases}

For this study we used two analytically defined test cases. The first test case is a region containing a bipole loop and the second is a region containing a unipolar magnetic field. For both test cases magnetic fields are calculated over a domain size of $n x=n y=n z=200$ with grid spacing of $d=0.01$. Defining the extends of our domain as $[-1: 1,-1: 1,0: 2]$ with its origin at $(0,0,0)$, the test cases can be defined in terms of the input magnetic field $B_{z 0}$ as follows:

Test Case 1. The formula for a bipole loop is determined by taking the analytic expression for a force-free bipole field given in Cuperman et al. (1989). We take the current free version of these equations and set the coordinates of the magnetic poles to $\left(x_{01}, y_{01}, z_{01}\right)=(-0.5,0,-1)$ and $\left(x_{02}, y_{02}, z_{02}\right)=(0.5,0,-1)$. Finally we take the $B_{z}$ component and set $z=0$ to arrive at

$B_{z 0}=A\left[\left(\frac{1}{\left((x+0.5)^{2}+y^{2}+1\right)^{3 / 2}}\right)-\left(\frac{1}{\left((x-0.5)^{2}+y^{2}+1\right)^{3 / 2}}\right)\right]$.

Test Case 2. The formula for a unipolar magnetic field is determined by assuming that the strength of the normal magnetic field $B_{z}$ drops off as a Gaussian with distance from a magnetic source

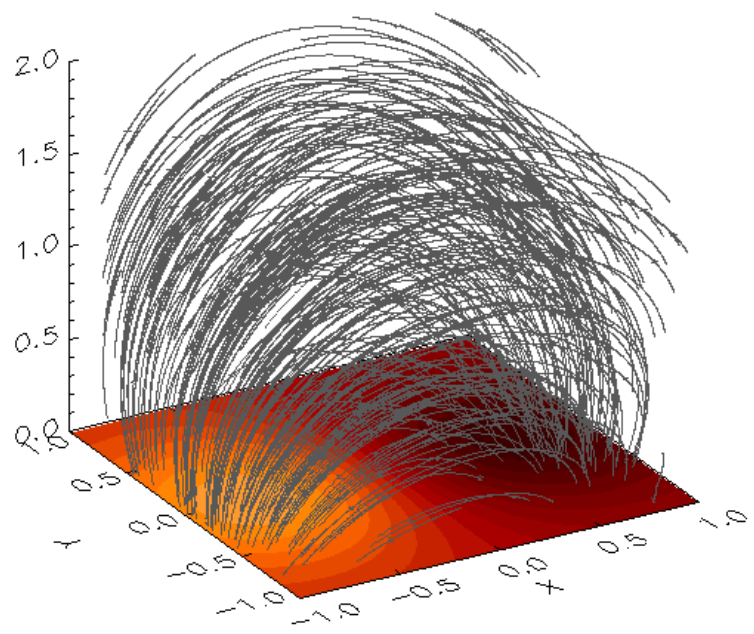

Fig. 2. Field line diagram of the magnetic field used for Test Case 1. The photospheric surface at the base of the domain is a coloured contour of $B_{z 0}$.

term. We take the position of the magnetic source term to be at the origin a unit distance beneath the photosphere to arrive at

$B_{z 0}=A \exp \left(-\frac{x^{2}+y^{2}+1}{2}\right)$,

The potential fields produced by these test cases are represented by field line diagrams in Figs. 2 and 3; the shading at the base of the domains in these images represents the strength and direction of the magnetic field at the photosphere.

\subsection{Metrics}

To analyse the magnetic fields that the potential field calculator produces we import each field into the MHD solver Lare3d and measure the current across the domain.

We then set the initial velocity to zero, the initial pressure to a constant value of $P=5 \times 10^{-9}$, and resistivity to $\eta=5 \times 10^{-5}$. We set $\gamma=5 / 3$ so the fluid is adiabatic and set the shock viscosities to $v_{1}=0.1$ and $v_{2}=0.5$. We set the boundary conditions such that the velocity and magnetic field have fixed values at the boundaries.

We then allow the simulation to evolve for $100 \tau_{A}$ where $\tau_{A}$ is the Alfvén time. The number of timesteps this requires varies as Lare3d uses a CFL limited timestep at each iteration. This is not intended as a relaxation phase but rather test of how stable the potential field configuration is over time. Finally we measure the current, velocity, and difference between the final and initial magnetic fields across the domain.

The metrics used to analyse the effectiveness of each method are given below. The closer these values are to zero the closer the field is to a potential field and the closer our equilibrium is to static equilibrium. The average current densities are the mean value of the current density $\mathbf{J}$ over all grid points. These metrics include

$\mathrm{MAJ}_{0}$ The maximum absolute current density at time zero.

$\mathrm{J}_{\mathrm{avg} 0} \quad$ The average absolute current density at time zero.

$\mathrm{MAJ}_{f}$ The maximum absolute current density after $100 \tau_{A}$.

$\mathrm{J}_{\mathrm{avg} f} \quad$ The average absolute current density after $100 \tau_{A}$.

MAV The maximum absolute velocity after $100 \tau_{A}$.

$\Delta B_{\max } \quad$ The maximum difference between initial and final magnetic fields. 


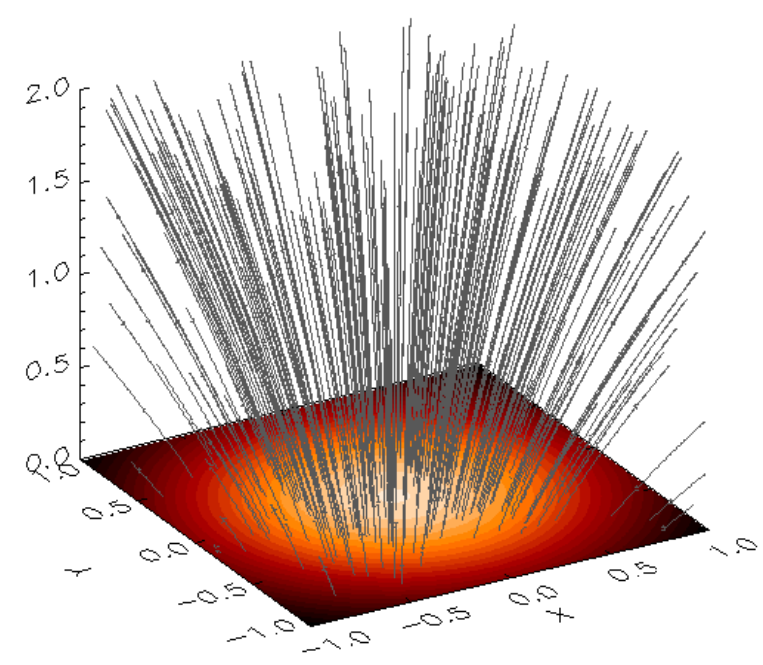

Fig. 3. Field line diagram of the magnetic field used for Test Case 2. The photospheric surface at the base of the domain is a coloured contour of $B_{z 0}$.

\section{Differentiation of magnetic potential $\phi$}

\subsection{Method}

In order to calculate the magnetic field components in our domain we need to differentiate the magnetic field potential according to Eq. (5). The effect of grid staggering is that the magnetic field component in a particular direction must be calculated at the cell edges in that direction not the cell centre. The magnetic potential $\phi$ however is calculated at the cell centres. This is illustrated in Fig. 1.

In this section three methods of numerical differentiation are presented, which each account differently for this discrepancy. The calculation of $B_{x}(i, j, k)$ is taken as an example. The indices $j$ and $k$ are taken to be fixed and $B_{x}(i, j, k)$ is denoted simply as $B_{x}^{i}$.

Method $A$. In this method a central difference formula of order $O\left(d^{4}\right)$ is used and the grid staggering is not taken into account. The magnetic field calculated using method $\mathrm{A}$ is denoted as $B_{x A}^{i}$. The formula used to calculate $B_{x A}^{i}$ is given in Eq. (11) below. This method requires an additional four ghost cells in each direction, i.e.

$B_{x A}^{i}=\frac{\phi^{i-2}-8 \phi^{i-1}+8 \phi^{i+1}-\phi^{i+2}}{12 d}$.

Method $B$. In this method the grid staggering is accounted for by calculating the magnetic field components at the cell centres using method $\mathrm{A}$ and then averaging these values to get the magnetic field components at the cell edges. The magnetic field calculated using method $\mathbf{B}$ is denoted as $B_{x B}^{i}$. The formula used to calculate $B_{x B}^{i}$ is given in Eq. (12) below. This method requires an additional five ghost cells in each direction, i.e.

$B_{x B}^{i}=\frac{B_{x A}^{i}+B_{x A}^{i+1}}{2}$.

Method $C$. In this method the grid staggering is accounted for by calculating the magnetic field components using a different central difference formula. A full derivation of this formula is given in Appendix A. The magnetic field calculated using method $\mathrm{C}$ is denoted as $B_{x C}^{i}$. The formula used to calculate $B_{x C}^{i}$
Table 1. Value of each metric for Method A, Method B, and Method C of numerical differentiation for Test Case 1 .

\begin{tabular}{|c|c|c|c|}
\hline \multicolumn{4}{|c|}{ Test Case 1} \\
\hline & Method A* & Method B & Method C \\
\hline $\mathrm{MAJ}_{0}$ & $4.45 \times 10^{-1}$ & $1.72 \times 10^{-1}$ & $7.86 \times 10^{-3}$ \\
\hline $\mathrm{J}_{\mathrm{avg} 0}$ & $2.81 \times 10^{-3}$ & $3.23 \times 10^{-5}$ & $1.61 \times 10^{-5}$ \\
\hline MAJ $_{f}$ & $3.79 \times 10^{-1}$ & $2.01 \times 10^{-2}$ & $1.97 \times 10^{-2}$ \\
\hline $\mathrm{J}_{\mathrm{avg} f}$ & $2.58 \times 10^{-3}$ & $1.16 \times 10^{-4}$ & $1.01 \times 10^{-4}$ \\
\hline MAV & $1.14 \times 10^{-2}$ & $2.27 \times 10^{-5}$ & $1.83 \times 10^{-5}$ \\
\hline$\Delta B_{\max }$ & $8.14 \times 10^{-3}$ & $7.02 \times 10^{-4}$ & $4.06 \times 10^{-4}$ \\
\hline
\end{tabular}

Notes. ${ }^{*}$ The values are for $50 \tau_{A}$ instead of $100 \tau_{A}$.

Table 2. Value of each metric for Method A, Method B, and Method C of numerical differentiation for Test Case 2.

\begin{tabular}{lccc}
\hline \hline \multicolumn{3}{c}{ Test Case 2 } \\
\hline & Method A & Method B & Method C \\
\hline MAJ $_{0}$ & $2.97 \times 10^{-1}$ & $1.15 \times 10^{-2}$ & $5.25 \times 10^{-3}$ \\
$\mathrm{~J}_{\text {avg0 }}$ & $1.97 \times 10^{-3}$ & $2.98 \times 10^{-5}$ & $1.48 \times 10^{-5}$ \\
MAJ $_{f}$ & $2.13 \times 10^{-1}$ & $1.40 \times 10^{-2}$ & $1.37 \times 10^{-2}$ \\
$\mathrm{~J}_{\text {avg } f}$ & $2.10 \times 10^{-3}$ & $7.58 \times 10^{-5}$ & $7.13 \times 10^{-5}$ \\
MAV & $3.38 \times 10^{-3}$ & $1.36 \times 10^{-5}$ & $1.12 \times 10^{-5}$ \\
$\Delta B_{\max }$ & $2.40 \times 10^{-3}$ & $2.82 \times 10^{-4}$ & $2.10 \times 10^{-4}$ \\
\hline
\end{tabular}

is given in Eq. (13) below. This method requires an additional three ghost cells in each direction, i.e.

$B_{x C}^{i}=\frac{\phi^{i-1}-27 \phi^{i}+27 \phi^{i+1}-\phi^{i+2}}{24 d}$

\subsection{Results}

For each method of differentiation we generated a potential field for each test case. The potential fields were generated from input fields $B_{z 0}$ defined over the base of our computational domain. We analysed and compared the potential fields generated and the results of our analysis are given in Tables 1 and 2 .

For Test Case 1 the potential field produced using Method 1 was not run to $100 \tau_{A}$. The currents present in the initial magnetic field caused $\nabla \cdot \mathbf{v}$ to increase, significantly reducing $\rho$ in some parts of the domain. This led to a significant reduction in the CFL limited timestep as the simulation progressed making it take unfeasibly long to run to a simulation time of $100 \tau_{A}$; the values given are instead for $50 \tau_{A}$.

From Tables 1 and 2 we can see that the magnetic field generated is closest to a potential field when Method C is used. Although the difference between using Methods B and C can be marginal Method $\mathrm{C}$ is the most accurate method of numerical differentiation. Furthermore Method $\mathrm{C}$ has the advantage of being more computationally efficient, as it does not require any averaging after the initial differentiation and requires the least number of additional ghost cells for the potential calculation. Method $\mathrm{C}$ was therefore selected as the preferred option for our potential calculator.

\section{Size of input region for $\boldsymbol{B}_{\mathbf{z} 0}$}

\subsection{Method}

We now consider the size of the input region, that is the area at the photospheric boundary over which the input field $B_{z 0}$ is 


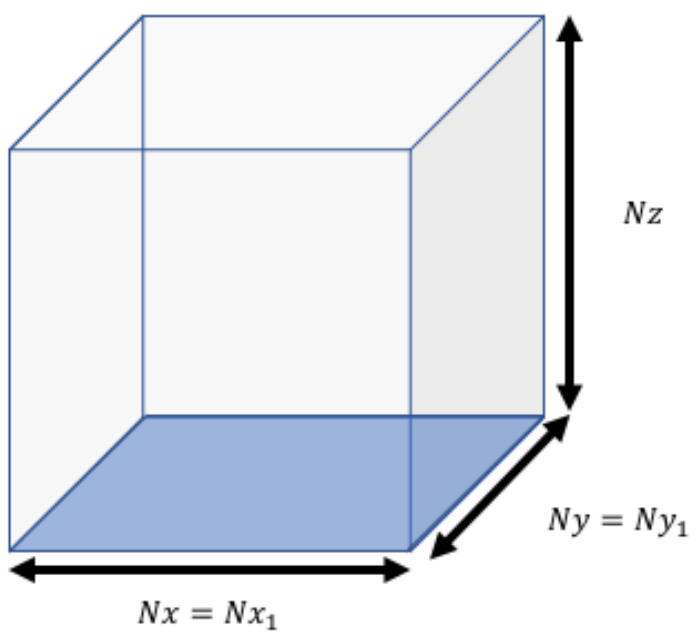

Fig. 4. llustration of the computational domain for $\phi$, shown as the grey box, and the input region for $B_{z 0}$, shown as the blue square, when $\left(N x_{1}, N y_{2}\right)$ is equal to $(N x, N y)$.

specified. The size of the computational domain for $\phi$ is denoted as $(N x, N y, N z)$ and the size of the input region for $B_{z 0}$ is denoted as $\left(N x_{1}, N y_{2}\right)$. So far the input field $B_{z 0}$ has only been defined over the base of our computational domain. In other words $\left(N x_{1}, N y_{2}\right)$ has been set to exactly equal $(N x, N y)$. The input field $B_{z 0}$ can however be defined over a larger area.

The motivation for extending our input region is that the current $J$ in our previous potential fields has been concentrated at the $x / y$ boundaries. The reason for this can be seen in Fig. 6; the gradient of the magnetic field strength changes suddenly at these boundaries. By extending the input region we attempt to reduce the effect of this change in gradient and reduce the current at the boundaries. We consider two options for the size of the input region:

Standard input region - $\left(N x_{1}, N y_{2}\right)$ is set to equal exactly $(N x, N y)$ and the centres of the input region and computational domain are aligned. The input data then covers exactly the same area of photosphere as the computational domain for $\phi$. This is illustrated in Fig. 4.

Extended input region - $\left(N x_{1}, N y_{2}\right)$ is set equal to $(3 N x, 3 N y)$ and the centres of the input region and computational domain are aligned. The input data then covers a much larger area of photosphere than the computational domain for $\phi$. This is illustrated in Fig. 5.

\subsection{Results}

For each test case we produce potential fields using both the standard and extended input regions. We used Eq. (6) to calculate our magnetic potential $\phi$ and Method $C$ to perform our numerical differentiation. The potential fields generated were each run in Lare $3 \mathrm{~d}$ for $100 \tau_{A}$ before being analysed and compared. The results of our analysis are given in Tables 3 and 4 . We can see that the magnetic field generated using a larger input region is closer to a potential field. Furthermore we can see from Figs. 6 and 7 that using a larger input region produces magnetic fields that are smoother at the boundaries.

It is worth noting that because of the nature of the Green's function method used the potential calculator expects $B_{z 0}$ to equal zero outside of the input region. If significant magnetic fields are expected outside of the input region then the accuracy of the calculator can be improved by extending the size of the

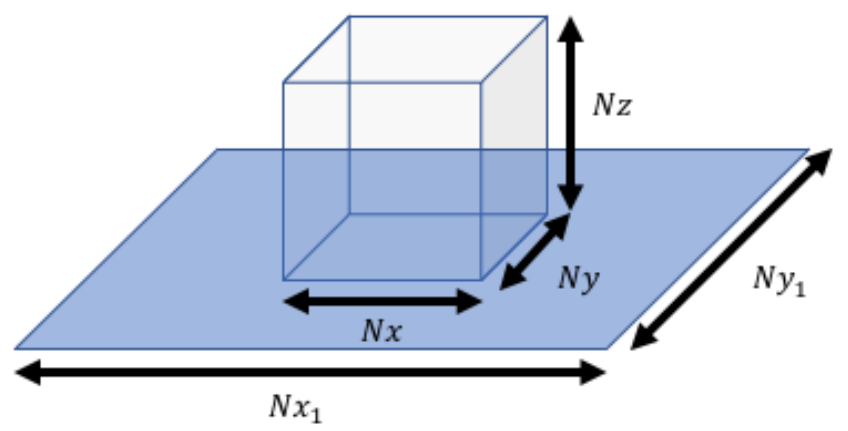

Fig. 5. Illustration of the computational domain for $\phi$, shown as the grey box, and the input region for $B_{z 0}$, shown as the blue square, when $\left(N x_{1}, N y_{2}\right)$ is equal to $(3 N x, 3 N y)$.

Table 3. Value of each metric using standard and extended input regions for Test Case 1.

\begin{tabular}{lcc}
\hline \hline \multicolumn{3}{c}{ Test Case 1 } \\
\hline & Standard & Extended \\
\hline MAJ $_{0}$ & $7.86 \times 10^{-3}$ & $1.01 \times 10^{-4}$ \\
$\mathrm{~J}_{\text {avg0 }}$ & $1.61 \times 10^{-5}$ & $6.42 \times 10^{-6}$ \\
MAJ $_{f}$ & $1.97 \times 10^{-2}$ & $1.90 \times 10^{-3}$ \\
$\mathrm{~J}_{\text {avg } f}$ & $1.01 \times 10^{-4}$ & $5.35 \times 10^{-5}$ \\
MAV & $1.83 \times 10^{-5}$ & $1.74 \times 10^{-5}$ \\
$\Delta B_{\max }$ & $4.06 \times 10^{-4}$ & $1.64 \times 10^{-4}$ \\
\hline
\end{tabular}

Table 4. Value of each metric using standard and extended input regions for Test Case 2.

\begin{tabular}{lcc}
\hline \hline \multicolumn{3}{c}{ Test Case 2 } \\
\hline & Standard & Extended \\
\hline MAJ $_{0}$ & $5.25 \times 10^{-3}$ & $7.99 \times 10^{-6}$ \\
$\mathrm{~J}_{\text {avg0 }}$ & $1.48 \times 10^{-5}$ & $1.72 \times 10^{-6}$ \\
MAJ $_{f}$ & $1.37 \times 10^{-2}$ & $5.64 \times 10^{-4}$ \\
$\mathrm{~J}_{\text {avg } f}$ & $7.13 \times 10^{-5}$ & $1.39 \times 10^{-5}$ \\
MAV & $1.12 \times 10^{-5}$ & $2.55 \times 10^{-7}$ \\
$\Delta B_{\max }$ & $2.10 \times 10^{-4}$ & $2.40 \times 10^{-5}$ \\
\hline
\end{tabular}

input region; however, there is a trade-off between accuracy and computing time, besides which limited photospheric data may be available.

\section{Comparison with analytical bipole loop}

\subsection{Method}

In Sect. 5.1 we determined the optimal method of numerical differentiation (Method C) and in Sect. 6.1 we saw that using an extended input region produces fields that are closer to a potential field solution. We now compare a potential field solution calculated with these improvements to an analytical solution for the same potential field.

Using our chosen method of differentiation and extended input region we apply our Green's method for extrapolation, as detailed in Sect. 3, to calculate a potential field. This field is produced using the normal photospheric field for a bipole loop, given as Test Case 1 in Sect. 4. We compare this field solution to the analytic solution for a potential bipole field given in 


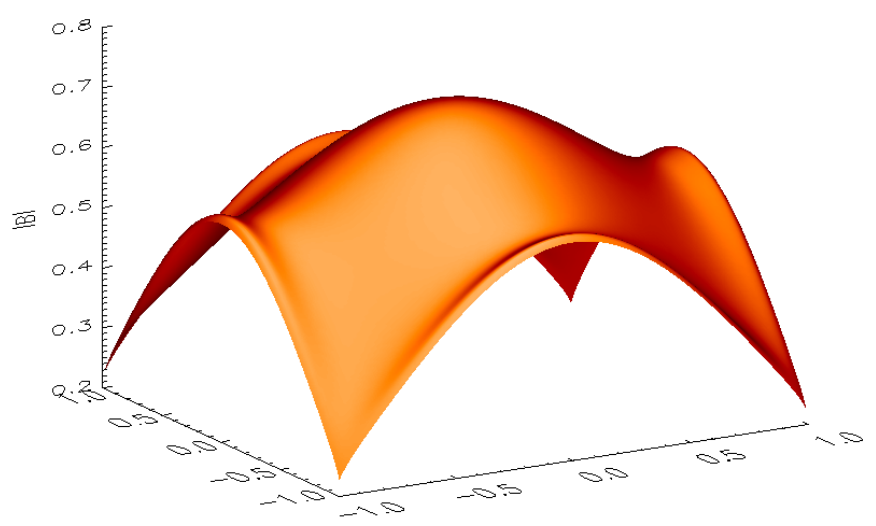

Fig. 6. Shaded surface diagram of the magnetic field strength at $z=0$ for the potential field generated using the standard input region. The flared edges are due to the discontinuity at the edge of the input region.

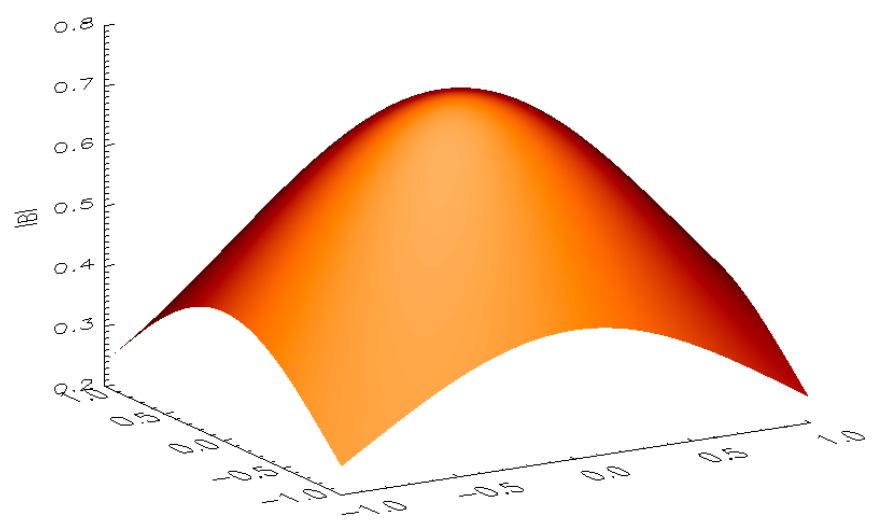

Fig. 7. Shaded surface diagram of the magnetic field strength at $z=0$ for the potential field generated using the extended input region. The edges are smoother because of the extended input region.

Cuperman et al. (1989), which has the same normal magnetic field at the photosphere. This is given by

$$
\begin{array}{llc}
B_{x}=B_{x, 1}-B_{x, 2}, & B_{y}=B_{y, 1}-B_{y, 2}, & B_{z}=B_{z, 1}-B_{z, 2}, \\
B_{x, j}=\frac{x_{j}}{R_{j}^{3}}, & B_{y, j}=\frac{y_{j}}{R_{j}^{3}}, & B_{z, j}=\frac{z_{j}}{R_{j}^{3}}, \\
R_{j}^{2}=x_{j}^{2}+y_{j}^{2}+z_{j}^{2}, & \\
x_{j}=x-x_{0, j}, & y_{j}=y-y_{0, j}, & z_{j}=z-z_{0, j},
\end{array}
$$

where $R_{j}$ are the distances from each magnetic pole for $j=1,2$ positioned at $\left(x_{01}, y_{01}, z_{01}\right)=(-0.5,0,-1)$ and $\left(x_{02}, y_{02}, z_{02}\right)=$ $(0.5,0,-1)$.

\subsection{Results}

The magnetic field produced by our potential calculator and the potential field given by Eq. (14) were both run in Lare3d and analysed in the same way as for the previous magnetic fields. This was done to compare both the initial magnetic fields and the evolution of these field after $100 \tau_{A}$ of simulation time in Lare $3 \mathrm{~d}$.

The results of this analysis are given in Table 5. The currents present in the analytical field can be explained by numerical diffusion. Numerical diffusion is caused by the central differencing scheme used to calculate the currents in Lare $3 \mathrm{~d}$. The truncation
Table 5. Value of each metric for the analytical and numerically calculated potential fields used for Test Case 1.

\begin{tabular}{lcc}
\hline \hline & Analytical & Numerical \\
\hline MAJ $_{0}$ & $1.20 \times 10^{-4}$ & $1.01 \times 10^{-4}$ \\
$\mathrm{~J}_{\text {avg0 }}$ & $7.54 \times 10^{-6}$ & $6.42 \times 10^{-6}$ \\
MAJ $_{f}$ & $2.18 \times 10^{-3}$ & $1.90 \times 10^{-3}$ \\
$\mathrm{~J}_{\text {avg } f}$ & $6.14 \times 10^{-5}$ & $5.35 \times 10^{-5}$ \\
MAV $^{-1.70 \times 10^{-5}}$ & $1.74 \times 10^{-5}$ \\
$\Delta B_{\max }$ & $2.32 \times 10^{-4}$ & $1.64 \times 10^{-4}$ \\
\hline
\end{tabular}

error or the central difference scheme used to calculate the currents is of the order $O\left(d^{2}\right)$, where h is the grid spacing. Indeed we can see that maximum current in this solution is also of the order $O\left(d^{2}\right)$ with grid spacing $d=0.01$.

These results show that the currents in the numerical solution as calculated by the potential calculator are similar and slightly smaller than those for the analytical solution. We can therefore say that, for this example, the numerical field solution of our potential calculator is correct to within the numerical diffusion of the simulation grid.

\section{Solar magnetogram data}

\subsection{Method}

Having determined the preferred methodology for our potential calculator and compared this to an analytical solution, we conclude our analysis by considering the magnetic field generated from solar magnetogram data. The data used was taken from line-of-sight magnetogram data collected by the Helioseismic and Magnetic Imager (HMI) instrument on the Solar Dynamics Observatory (SDO) and accessed through the Joint Science Operations Center (JSOC) on-line database. The data was taken from an active region close to the centre of the solar disc between $14: 02$ and $14: 56$ on $31 / 08 / 2003$. As the data was taken from a small area in the centre disc, it was taken to approximately represent the normal magnetic field data in that area. The data was normalised so that the maximum field strength was one and therefore the results are comparable with those from earlier sections.

A contour plot of the magnetogram data is given in Fig. 8 and the potential field produced using this magnetogram data is represented by a field line diagram shown in Fig. 9. Using this data potential fields were produced using each of the three methods of numerical differentiation described in Sect. 5.1 for a standard input region, as described in Sect. 6.1, and using our preferred Method $\mathrm{C}$ for numerical differentiation for an extended input region.

\subsection{Results}

The potential fields produced were run in Lare $3 \mathrm{~d}$ and analysed in the same way as for the previous magnetic fields. The potential field produced using Method A for numerical differentiation, which ignores the effect of the staggered grid, would not run for even $5 \tau_{A}$ and had initial maximum currents an order of magnitude larger than any of the other potential fields. The results for the analysis of the remaining potential fields are given in Table 6.

Comparing the results for using Method B or Method C for numerical differentiation, we can see that, consistent with 


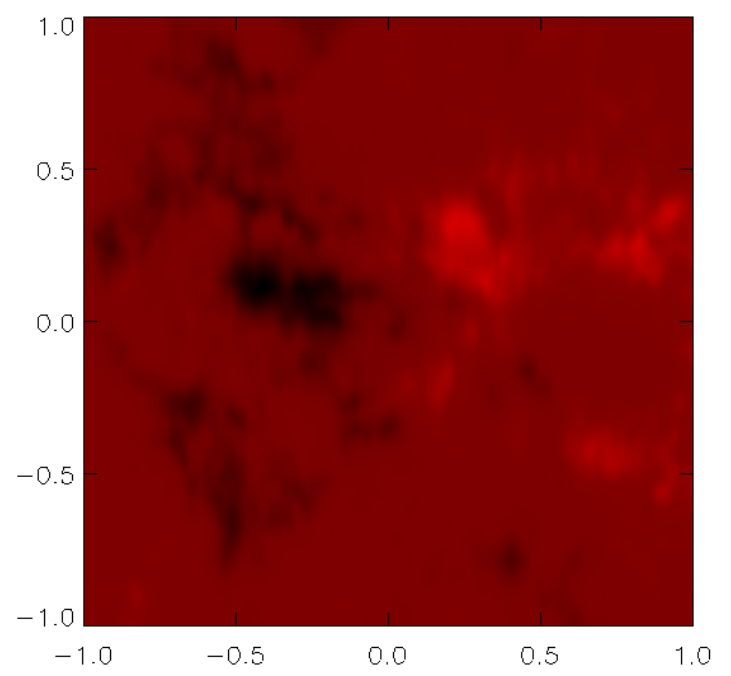

Fig. 8. Contour plot of the original magnetogram data of the normal magnetic field at the photosphere. The colour indicates the direction and strength of the magnetic field.

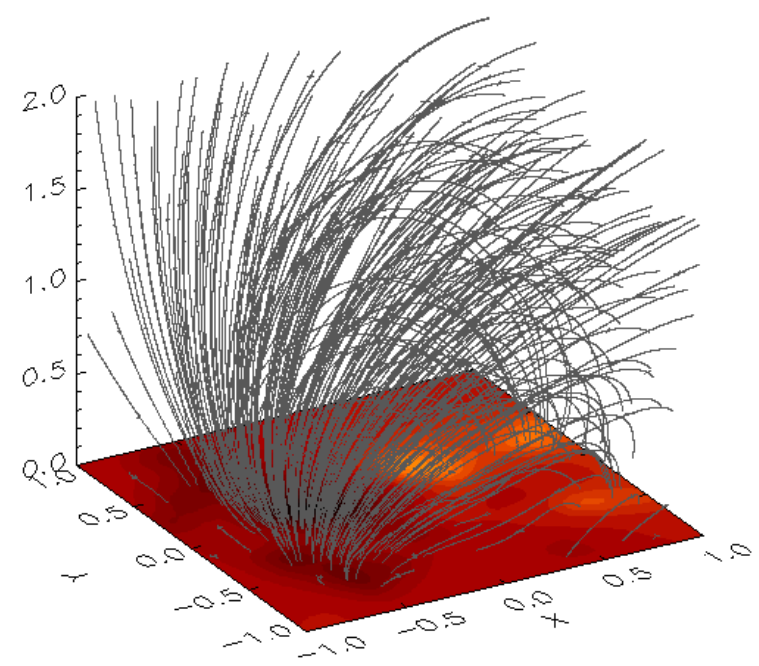

Fig. 9. Field line diagram of the potential magnetic field produced using solar magnetogram data. The photospheric surface at the base of the domain is a coloured contour of $B_{z 0}$.

our earlier results, Method C produces lower initial currents. Although over time the fields approach similar current values, the field produced using Method C maintains lower currents, changes less over time, and results in a more static equilibrium. This result supports our conclusion that Method $\mathrm{C}$ is the more accurate method in addition to being computationally quicker and requiring less ghost cells.

Comparing now the results for using either a standard or extended input region (both using Method $\mathrm{C}$ for differentiation) we see a surprising result. The maximum current is identical in both cases and the average current is marginally lower when the standard input region is used. Although the maximum velocity at $100 \tau_{A}$ is lower when the extended input region is used this difference is less than an order of magnitude. The reason is that using an extended input region has little effect, which can clearly be seen by comparing the magnetic field strength for the potential field generated with the standard input region and extended input region. The field strength at the photosphere is shown for each of these fields in Figs. 10 and 11. It can clearly be seen that
Table 6. Value of each metric for each of the potential fields produced using the solar magnetogram data.

\begin{tabular}{lccc}
\hline \hline & $\begin{array}{c}\text { Method B } \\
\text { (standard input) }\end{array}$ & $\begin{array}{c}\text { Method C } \\
\text { (standard input) }\end{array}$ & $\begin{array}{c}\text { Method C } \\
\text { (extended input) }\end{array}$ \\
\hline MAJ $_{0}$ & $2.36 \times 10^{-2}$ & $1.23 \times 10^{-2}$ & $1.23 \times 10^{-2}$ \\
$\mathrm{~J}_{\text {avg0 }}$ & $1.55 \times 10^{-5}$ & $7.88 \times 10^{-6}$ & $7.90 \times 10^{-6}$ \\
MAJ $_{f}$ & $4.92 \times 10^{-2}$ & $4.90 \times 10^{-2}$ & $4.90 \times 10^{-2}$ \\
$\mathrm{~J}_{\text {avg } f}$ & $4.45 \times 10^{-5}$ & $3.98 \times 10^{-5}$ & $4.03 \times 10^{-5}$ \\
MAV & $1.95 \times 10^{-3}$ & $1.81 \times 10^{-3}$ & $1.34 \times 10^{-3}$ \\
$\Delta B_{\max }$ & $4.14 \times 10^{-3}$ & $1.91 \times 10^{-3}$ & $1.91 \times 10^{-3}$ \\
\hline
\end{tabular}

in contrast to Fig. 7 in Sect. 6.1 the field strength in Fig. 11 is much smaller and flatter at the boundaries so assuming $B_{z 0}=0$ outside of the domain has much less of an effect.

Although extending the input region in general improves the accuracy of the potential field solution, these results indicate that the benefits of extending the input region size are sometimes only marginal, depending on the input field $B_{z 0}$. It must be remembered that to achieve the slight reduction in maximum velocity at $100 \tau_{A}$, the input region had to be extended to roughly nine times. This means that nine times more initial data is required and it also means that the runtime will increase ninefold.

\section{Conclusion}

A potential field calculator has been designed to accurately calculate a potential magnetic field over a domain given the normal field $B_{z 0}$ at the photospheric boundary. The design of the code was aimed at increasing accuracy by taking into consideration: the calculation of magnetic potential $\phi$, the differentiation of magnetic potential $\phi$, and the size of input region for $B_{z 0}$ as described in Sects. 2, 5.1, and 6.1 of this paper, respectively.

The potential field calculator uses Eq. (6) to calculate the magnetic potential. The potential is then differentiated using a novel central difference formula Eq. (13), derived in Appendix A, to calculate the magnetic field components. It has been shown that ignoring the staggered magnetic fields during differentiation of the magnetic potential results in an unstable potential field and that, whilst simple interpolation can be effective, the chosen method of numerical differentiation is ultimately more accurate, computationally quicker, and requires less ghost cells to perform. Further it has been shown in Sect. 7.1 that for analytically specified input data, this method can produce potential fields that are correct to within the numerical diffusion of the simulation grid.

The size of the input region, over which the normal magnetic field at the photosphere $B_{z 0}$ is specified, has been shown to effect the accuracy of the potential fields generated. It has been shown that in some cases extending the input region dramatically improves the accuracy of the potential field solution whilst in other cases the benefits of extending the input region size are only marginal. In all cases however extending the input region requires more input data to be specified and increases the runtime of the potential calculator. Depending on the improvement to the potential field solution, the availability of photospheric data, and the desired runtime of the calculator, it may or may not be beneficial to use an extended input region. The potential field calculator therefore leaves the extension of the input region as an option to the user. 


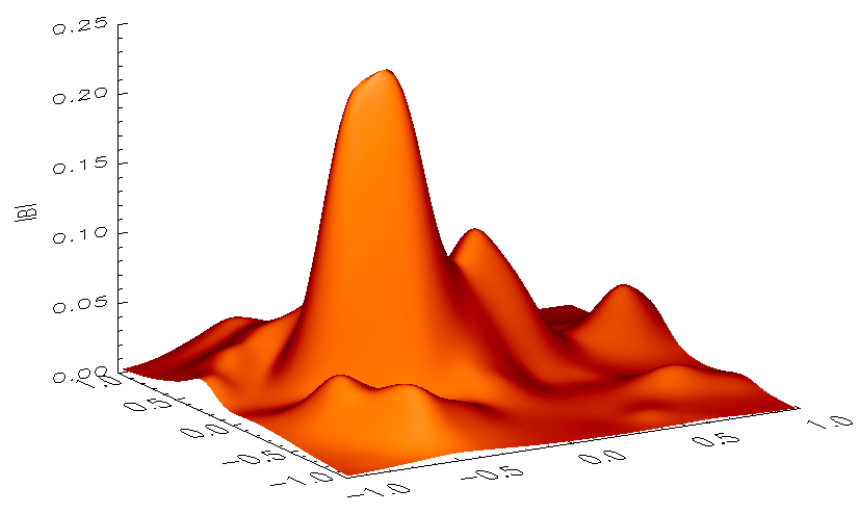

Fig. 10. Shaded surface diagram of the magnetic field strength at $z=0$ for the potential field generated using solar magnetogram data and a standard input region.

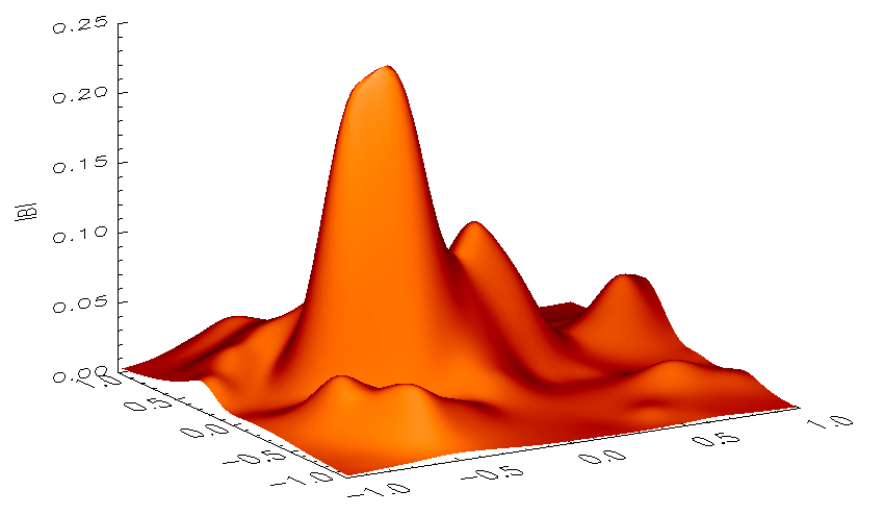

Fig. 11. Shaded surface diagram of the magnetic field strength at $z=0$ for the potential field generated using solar magnetogram data and an extended input region.

It should be noted that the equilibria produced using the potential fields generated may be further improved within an MHD solver by applying a phase of numerical relaxation. In Lare $3 \mathrm{~d}$ this is often done by artificially increasing the resistivity and allowing the system to relax before a simulation is run properly.

Whilst the Green's function methods used in this work have been available for a considerable length of time (Schmidt 1964; Semel 1967; Sakurai 1982), it is hoped that this accurate and simple implementation will be of considerable utility to those working with potential fields on a staggered grid. The potential calculator code is written in FORTRAN 90 and has been designed to produce initial magnetic field inputs for Lare3d but will work with any MHD solver that uses a staggered grid for the magnetic field components. The program can be parallelised to run quickly over multiple computing cores. This code and supporting documentation are provided alongside this paper.
Acknowledgements. Callum Boocock would like to thank UK STFC DISCnet for financial support of his $\mathrm{PhD}$ studentship. David Tsiklauri's research as supported by Georgia's Shota Rustaveli National Science Foundation under Collaborative Research Project Call (compatriots call) 2016-2019. This research utilised Queen Mary's Apocrita HPC facility, supported by QMUL Research-IT (King et al. 2017).

\section{References}

Arber, T., Longbottom, A., Gerrard, C., \& Milne, A. 2001, J. Comput. Phys., 171,151

Arregui, I. 2015, Philos. Trans. R. Soc. London Ser. A, 373, 20140261

Aschwanden, M. J. 2004, Physics of the Solar Corona. An Introduction (Praxis Publishing Ltd)

Balsara, D. S., \& Kim, J. 2004, ApJ, 602, 1079

Beckers, J. M. 1971, in Solar Magnetic Fields, ed. R. Howard, IAU Symp., 43, 3 Bingert, S., \& Peter, H. 2011, A\&A, 530, A112

Bingert, S., \& Peter, H. 2013, A\&A, 550, A30

Bourdin, P. A., Bingert, S., \& Peter, H. 2013, A\&A, 555, A123

Browning, P. K., Gerrard, C., Hood, A. W., Kevis, R., \& van der Linden, R. A. M. 2008, A\&A, 485, 837

Cuperman, S., Ofman, L., \& Semel, M. 1989, A\&A, 216, 265

Gary, G. A. 1989, ApJS, 69, 323

Gent, F. A., Fedun, V., Mumford, S. J., \& Erdélyi, R. 2013, MNRAS, 435, 689

Goossens, M. 2003, An Introduction to Plasma Astrophysics and Magnetohydrodynamics (Springer), 294

Gordovskyy, M., Browning, P. K., Kontar, E. P., \& Bian, N. H. 2014, A\&A, 561, A72

Gudiksen, B. V., \& Nordlund, A. 2005, ApJ, 618, 1020

Inoue, S. 2016, Progress in Earth and Planetary Science, 3, 19

Inoue, S., Magara, T., \& Pandey, V. 2013, ApJ, 780, 101

Khomenko, E., \& Collados, M. 2006, ApJ, 653, 739

King, T., Butcher, S., \& Zalewski, L. 2017, Apocrita - High Performance Computing Cluster for Queen Mary University of London, DOI 10.5281/zenodo. 438045

Klimchuk, J. A. 2015, Philos. Trans. R. Soc. London Ser. A, 373, 20140256

Masson, S., Pariat, E., Aulanier, G., \& Schrijver, C. J. 2009, ApJ, 700, 559

Neukirch, T. 2005, in Chromospheric and Coronal Magnetic Fields, eds. D. E. Innes, A. Lagg, \& S. A. Solanki, ESA Spec. Publ., 596, 12.1

Ofman, L., \& Selwa, M. 2008, Proc. Int. Astron. Union, 4, 151

Oliver, R., Murawski, K., \& Ballester, J. L. 1998, A\&A, 330, 726

Otto, A., Büchner, J., \& Nikutowski, B. 2007, A\&A, 468, 313

Parnell, C. E., \& De Moortel, I. 2012, Philos. Trans. R. Soc. London Ser. A, 370, 3217

Peter, H., Warnecke, J., Chitta, L. P., \& Cameron, R. H. 2015, A\&A, 584, A68

Petrukhin, N., Ruderman, M., \& Didenkulova, E. 2018, MNRAS, 474, 2289

Pizzo, V. J. 1986, ApJ, 302, 785

Ruan, P., Wiegelmann, T., Inhester, B., et al. 2008, A\&A, 481, 827

Sakurai, T. 1982, Sol. Phys., 76, 301

Schmidt, H. U. 1964, NASA Spec. Publ., 50, 107

Semel, M. 1967, Ann. Astrophys., 30, 513

Smith, P. D., Tsiklauri, D., \& Ruderman, M. S. 2007, A\&A, 475, 111

Solanki, S. K., \& Steiner, O. 1990, A\&A, 234, 519

Tadesse, T., Wiegelmann, T., Gosain, S., MacNeice, P., \& Pevtsov, A. A. 2014 A\&A, 562, A105

Thackray, H., \& Jain, R. 2017, A\&A, 608, A108

Wiegelmann, T. 2004, Sol. Phys., 219, 87

Wiegelmann, T. 2007, Sol. Phys., 240, 227

Wiegelmann, T. 2008, J. Geophys. Res.: Space Phys., 113, A3

Wiegelmann, T., \& Neukirch, T. 2006, A\&A, 457, 1053

Wiegelmann, T., \& Sakurai, T. 2012, Liv. Rev. Sol. Phys., 9, 5

Wiegelmann, T., Neukirch, T., Ruan, P., \& Inhester, B. 2007, A\&A, 475, 701 


\section{Appendix A: Derivation of formula for numerical differentiation}

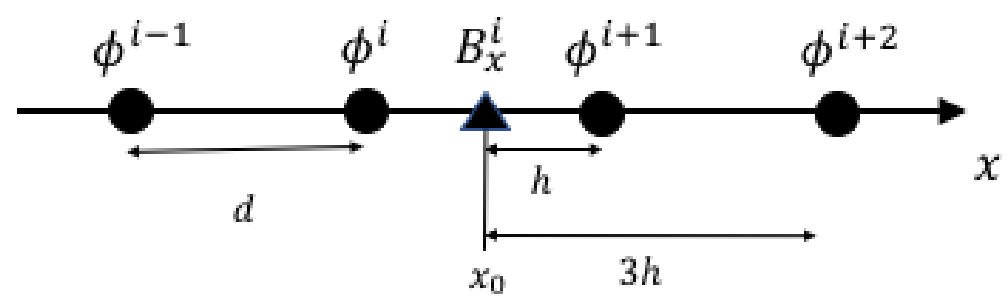

Fig. A.1. One-dimensional representation of a small section around $B_{x}^{i}$ at a point $x_{0}$ in the $x$ direction showing the position of $\phi$ values relative to $x_{0}$ in the $x$ direction.

Our potential field calculator calculates the magnetic field components by taking derivates of the magnetic potential according to Eq. (5) of the main text. The method of numerical differentiation used is a central difference formula of order $O\left(h^{4}\right)$ that has been adapted to work on a staggered grid. In this Appendix we derive this formula.

We take the calculation of $B_{x}(i, j, k)$ as an example, the indices $j$ and $k$ are taken to be fixed and $B_{x}(i, j, k)$ is denoted simply as $B_{x}^{i}$. We now consider the position of $B_{x}^{i}$ relative to the points, where $\phi$ is defined along the $x$-axis. If we define $d$ as the grid spacing and $h$ as half the grid spacing, then for $B_{x}^{i}$ defined at a point $x_{0}, \phi$ is defined at distances $h$ and $3 h$ either side of $x_{0}$. This is illustrated in Fig. A.1. Now we calculate the derivative of $\phi$ with respect to $x$ at the point $x_{0}$ that is $\phi^{\prime}\left(x_{0}\right)$. We begin by taking the Taylor expansions of $\phi^{i-1}, \phi^{i}, \phi^{i+1}$, and $\phi^{i+2}$ about $x$ these are given below in Eqs. (A.1)-(A.4) as follows:

$\phi^{i-1}=\phi\left(x_{0}-3 h\right)=\phi\left(x_{0}\right)-3 h \phi^{\prime}\left(x_{0}\right)+\frac{9 h^{2}}{2} \phi^{\prime \prime}\left(x_{0}\right)-\frac{27 h^{3}}{6} \phi^{\prime \prime \prime}\left(x_{0}\right)+O\left(h^{4}\right)$,

$\phi^{i}=\phi\left(x_{0}-h\right)=\phi\left(x_{0}\right)-h \phi^{\prime}\left(x_{0}\right)+\frac{h^{2}}{2} \phi^{\prime \prime}\left(x_{0}\right)-\frac{h^{3}}{6} \phi^{\prime \prime \prime}\left(x_{0}\right)+O\left(h^{4}\right)$,

$\phi^{i+1}=\phi\left(x_{0}+h\right)=\phi\left(x_{0}\right)+h \phi^{\prime}\left(x_{0}\right)+\frac{h^{2}}{2} \phi^{\prime \prime}\left(x_{0}\right)+\frac{h^{3}}{6} \phi^{\prime \prime \prime}\left(x_{0}\right)+O\left(h^{4}\right)$,

$\phi^{i+2}=\phi\left(x_{0}+3 h\right)=\phi\left(x_{0}\right)+3 h \phi^{\prime}\left(x_{0}\right)+\frac{9 h^{2}}{2} \phi^{\prime \prime}\left(x_{0}\right)+\frac{27 h^{3}}{6} \phi^{\prime \prime \prime}\left(x_{0}\right)+O\left(h^{4}\right)$.

First we eliminate the even powers of $h$ by taking $\phi^{i+2}-\phi^{i-1}$ and $\phi^{i+1}-\phi^{i}$ as shown below in Eqs. (A.5) and (A.6):

$\phi^{i+2}-\phi^{i-1}=6 h \phi^{\prime}\left(x_{0}\right)+9 h^{3} \phi^{\prime \prime \prime}\left(x_{0}\right)+O\left(h^{4}\right)$,

$\phi^{i+1}-\phi^{i}=2 h \phi^{\prime}\left(x_{0}\right)+\frac{h^{3}}{3} \phi^{\prime \prime \prime}\left(x_{0}\right)+O\left(h^{4}\right)$.

We then need to remove the third order term by taking $27\left(\phi^{i+1}-\phi^{i}\right)-\left(\phi^{i+2}-\phi^{i-1}\right)$ as shown below in Eq. (A.7):

$27\left(\phi^{i+1}-\phi^{i}\right)-\left(\phi^{i+2}-\phi^{i-1}\right)=48 h \phi^{\prime}\left(x_{0}\right)+O\left(h^{4}\right)$.

Rearranging this equation we have the central difference formula,

$B_{x}^{i}=\phi^{\prime}\left(x_{0}\right)=\frac{\phi^{i-1}-27 \phi^{i}+27 \phi^{i+1}-\phi^{i+2}}{48 h}$.

\section{Appendix B: Potential calculator - BooTsik.f90}

This Appendix provides the FORTRAN 90 code for the potential calculator, BooTsik.f90, which can also be found at https: //github.com/calboo/Potential_Field_Calculator. The user must set the domain size, $n x, n y, n z$; the grid spacing, $d$; the size of the input region, $n x 0, n x 1, n y 0, n y 1$, and must specify the normal field $B_{z 0}$ at the photosphere. There are preset options for the input fields $B_{z 0}$ used in this paper and for importing an input field from an unformatted data file that can be used simply by uncommenting them. The code is given below:

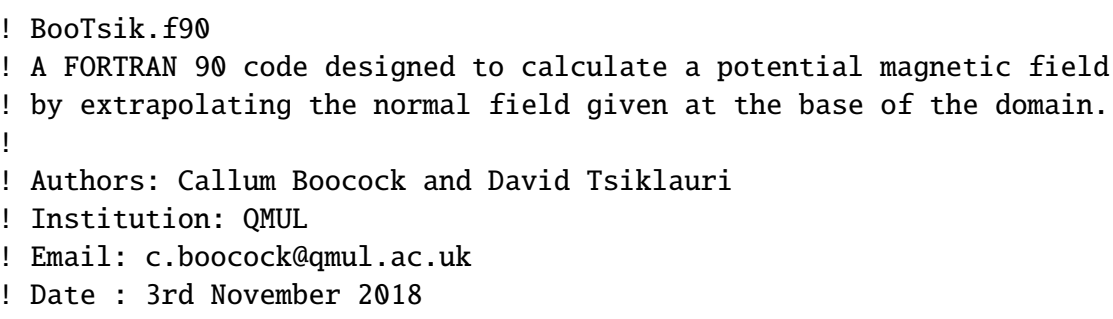




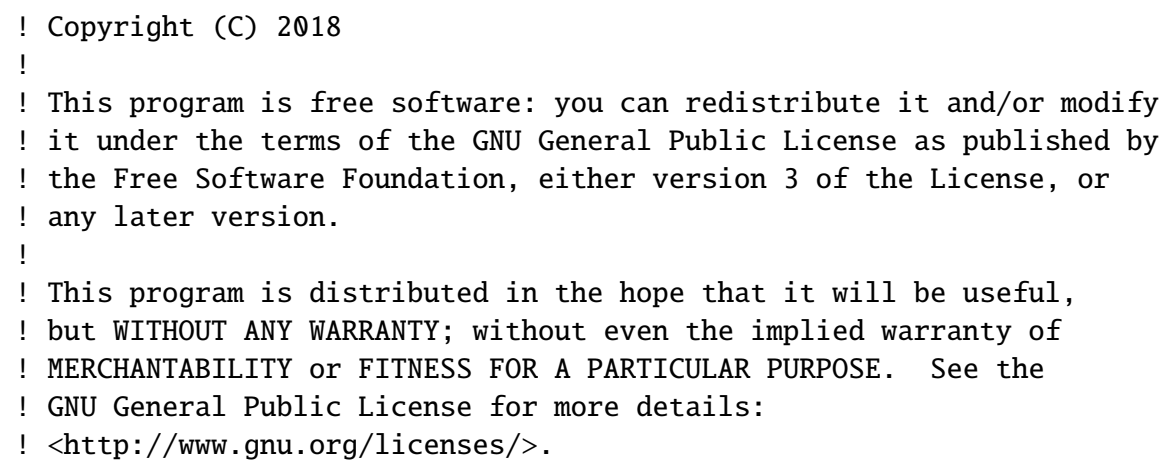




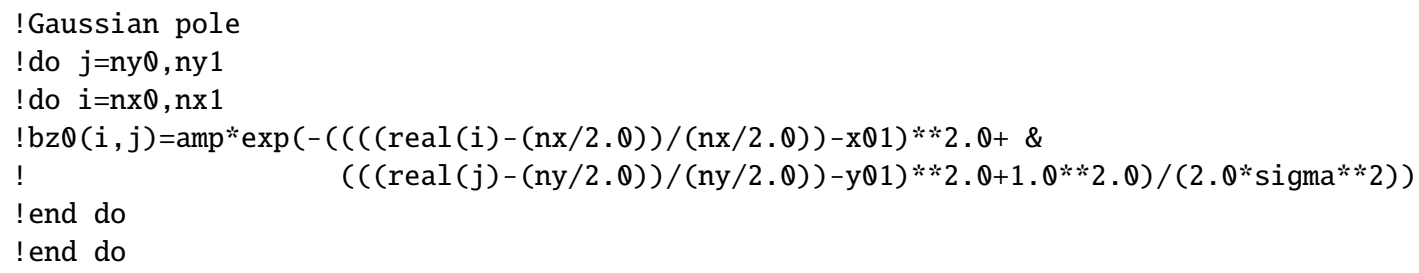




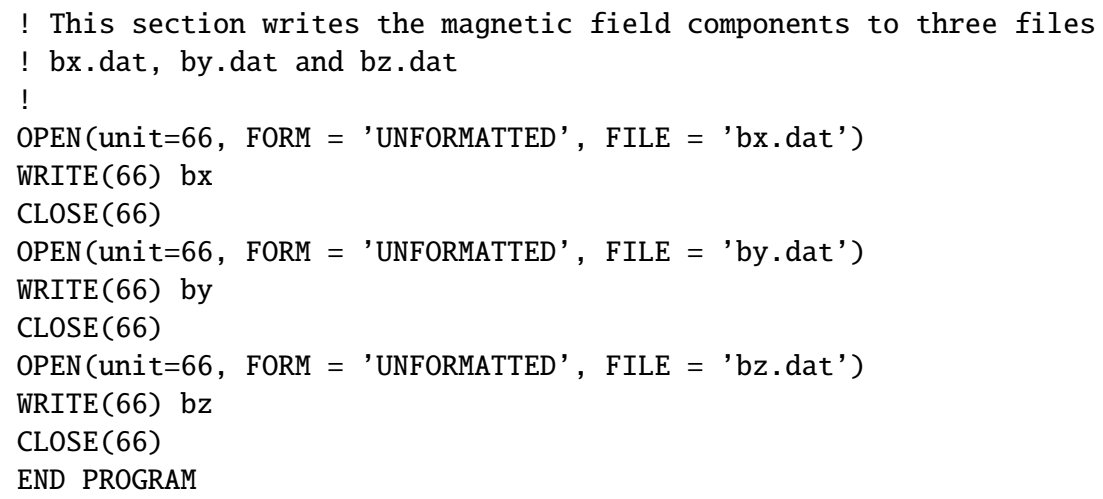

This code can be run in parallel on a HPC cluster. To achieve this the code can be auto-parallelised, using for example, an intel Fortran compiler. Using an Intel Fortran compiler the following line compiles the code in parallel ready to be submitted as a job on a HPC node. This significantly improves the runtime, depending on the number of cores used. The line is written as

ifort -o potential -03 -parallel potential.f90 -xHost.

\section{Appendix C: IDL visualisation tool - Boobox.pro}

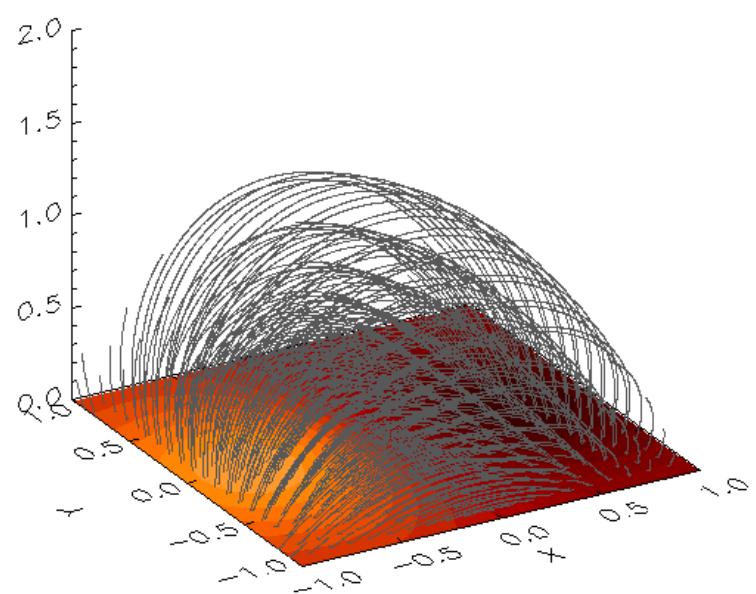

Fig. C.1. Field line diagram of a potential bipole field visualised with Boobox.pro with GRID=1. We can clearly see the magnetic field lines connected to each point at the photosphere.

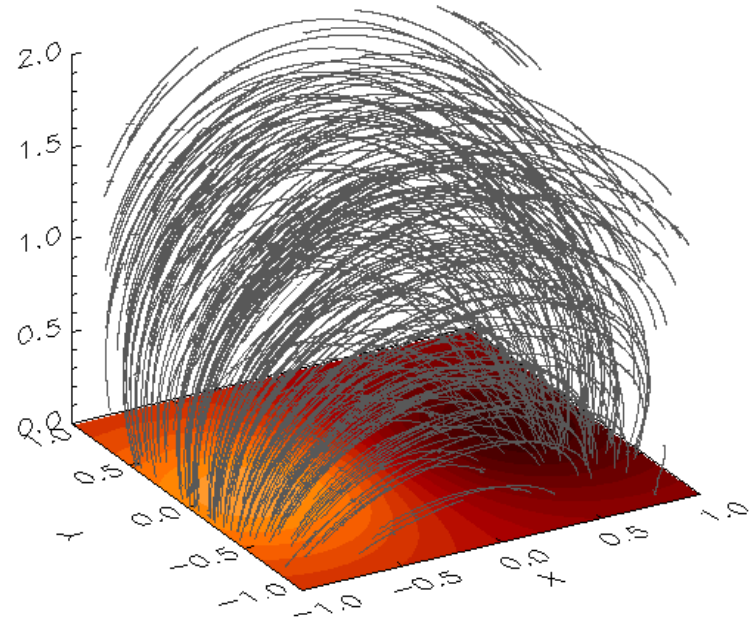

Fig. C.2. Field line diagram of a potential bipole field visualised with Boobox.pro with GRID=0. We can clearly see that the field strength is greater closer to the poles at the photosphere.

This appendix provides the IDL script for a visualisation tool, Boobox.pro, for visualising magnetic fields either from unstructured data files or from data snapshots from Lare 3d. The fields are visualised as field lines in a 3D box with the base of the box contoured according to the strength and direction of Bz at that boundary. The script was used to generate the field line images in this paper.

To use the tool the user must specify the domain dimensions, $n x, n y, n z$; the domain extents for the tick labels and the tick label format; the angles at which to visualise the domain, $a x, a z$, and must finally specify a value for the variable GRID. If GRID is set to the integer value 1 then the field lines are drawn from starting points uniformly distributed across the photospheric surface at the base of the domain. If GRID is set to any other value then the field lines are drawn from starting points randomly distributed throughout the domain, producing a plot where the density of the field lines corresponds roughly to the field strength. Examples of plots produced for a bipole loop using Boobox.pro with GRID set to 1 and 0 are given in Figs. C.1 and C.2.

If the tool is being used with unstructured data files of the magnetic field components bx.dat, by.dat, and bz.dat, then the appropriate block must be uncommented and the tool can be run in IDL with . $r$ boobox. If the tool is being used to visualise data from a Lare $3 \mathrm{~d}$ data snapshot, ds, then the first line pro boobox, ds, and the appropriate block must be uncommented and the tool can be run in IDL with boobox, ds, where ds is the data snapshot. The IDL script for Boobox.pro is given below:

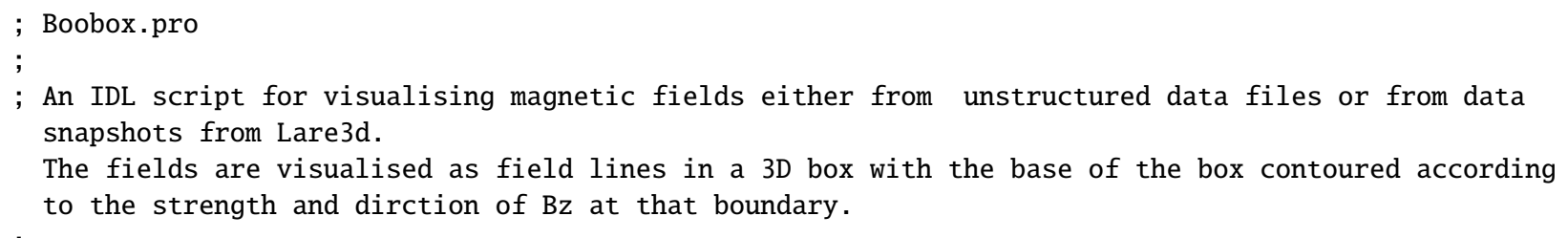

; 
C. M. Boocock and D. Tsiklauri: Potential field calculator for staggered magnetic grids

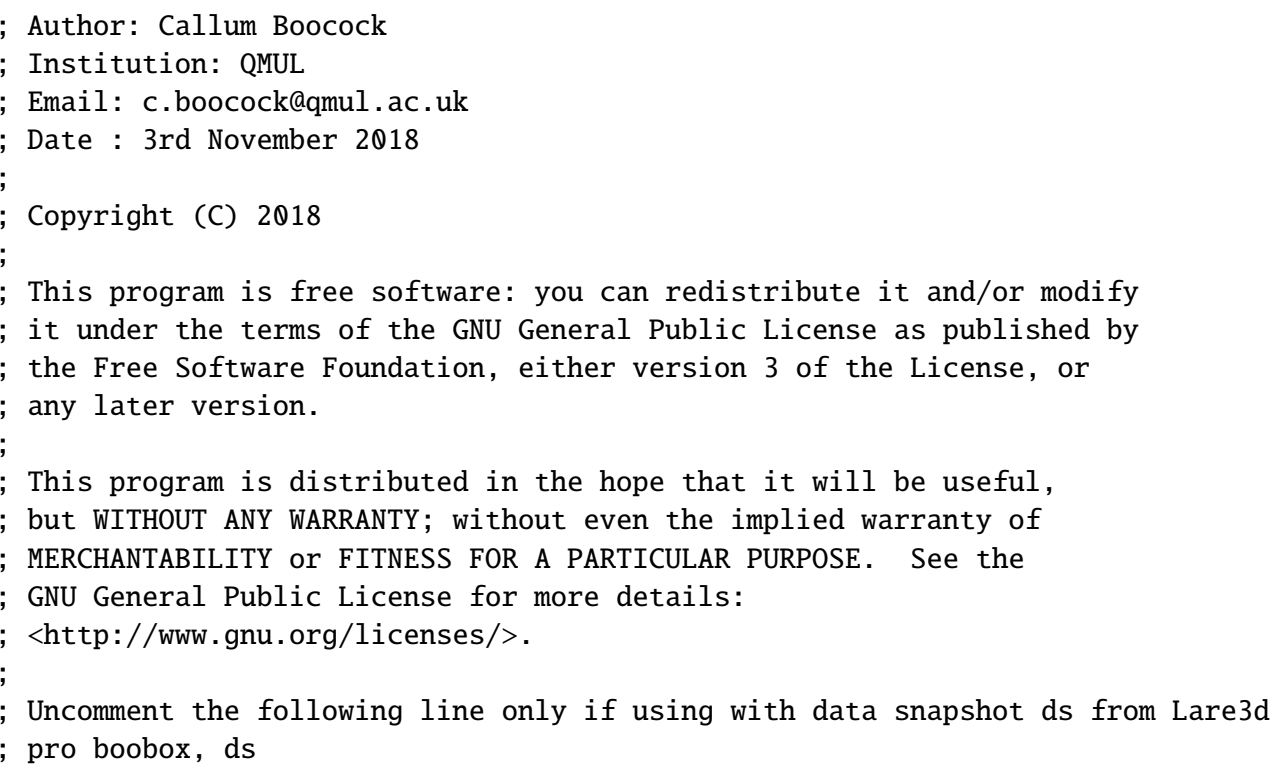


$; b z \Theta=b z \otimes(2: n x+2,2: n y+2,2: n z+3)$

; Uncomment this block for use with data snapshot ds from Lare3d

$; \mathrm{bx} \theta=\mathrm{ds} \cdot \mathrm{bx}$

; by $0=$ ds.by

$; \mathrm{bzQ}=\mathrm{ds} . \mathrm{bz}$

$; \mathrm{x}=\mathrm{ds} . \mathrm{x}$

$; y=d s \cdot y$

$; \mathrm{z}=\mathrm{ds} . \mathrm{z}$

; Calculate and format the tick labels

$\mathrm{x} 2=(\mathrm{xmin}+\mathrm{xmax}) / 2.0$

$\mathrm{x} 1=(\mathrm{xmin}+\mathrm{x} 2) / 2.0$

$\mathrm{x} 3=(\mathrm{x} 2+\mathrm{xmax}) / 2.0$

y2 $=(y \min +\mathrm{ymax}) / 2.0$

$\mathrm{y} 1=(\mathrm{ymin}+\mathrm{y} 2) / 2.0$

$\mathrm{y} 3=(\mathrm{y} 2+\mathrm{ymax}) / 2.0$

$\mathrm{z} 2=(\mathrm{zmin}+\mathrm{zmax}) / 2.0$

$\mathrm{z} 1=(\mathrm{zmin}+\mathrm{z} 2) / 2.0$

$z 3=(z 2+z \max ) / 2.0$

xmin = string (xmin, FORMAT $=$ tikform)

$\mathrm{x} 2=\operatorname{string}(\mathrm{x} 2, \mathrm{FORMAT}=$ tikform $)$

$\mathrm{x} 1=\operatorname{string}(\mathrm{x} 1, \mathrm{FORMAT}=\mathrm{tikform})$

$\mathrm{x} 3$ = string ( $\mathrm{x} 3$, FORMAT $=$ tikform $)$

xmax $=\operatorname{string}(x \max$, FORMAT $=$ tikform $)$

ymin $=\operatorname{string}(y m i n$, FORMAT $=$ tikform $)$

$\mathrm{y} 2$ = $\operatorname{string}(\mathrm{y} 2$, FORMAT=tikform $)$

$\mathrm{y} 1=\operatorname{string}(\mathrm{y} 1$, FORMAT=tikform $)$

y3 = string ( $\mathrm{y} 3$, FORMAT $=$ tikform $)$

ymax $=$ string (ymax, FORMAT=tikform)

zmin = string (zmin, FORMAT $=$ tikform)

$\mathrm{z2}=\operatorname{string}(\mathrm{z} 2$, FORMAT $=$ tikform $)$

$z 1=\operatorname{string}(z 1$, FORMAT $=$ tikform $)$

z3 = string ( $z 3$, FORMAT $=$ tikform $)$

zmax $=\operatorname{string}(z \operatorname{zmax}$, FORMAT $=$ tikform $)$

$\mathrm{xt}=[\operatorname{strtrim}(x \operatorname{xin}, 2), \operatorname{strtrim}(\mathrm{x} 1,2), \operatorname{strtrim}(\mathrm{x} 2,2), \operatorname{strtrim}(\mathrm{x} 3,2), \operatorname{strtrim}(x m a x, 2)]$

$\mathrm{yt}=[\operatorname{strtrim}(y \min , 2), \operatorname{strtrim}(y 1,2), \operatorname{strtrim}(y 2,2), \operatorname{strtrim}(y 3,2), \operatorname{strtrim}(y m a x, 2)]$

$\mathrm{zt}=[\operatorname{strtrim}(z \min , 2), \operatorname{strtrim}(z 1,2), \operatorname{strtrim}(z 2,2), \operatorname{strtrim}(z 3,2), \operatorname{strtrim}(z m a x, 2)]$

; Set colours

TVLCT, 255, 255, 255, 254 ; White color

TVLCT, $0,0,0,253 \quad$; Black color

TVLCT, $88,88,88,252$; Line color

!P.Color $=253$

!P. Background $=254$

; Clear window

WINDOW, 1, XSIZE $=600$, YSIZE $=500$, TITLE=' Boobox'

ERASE

; Set up the 3D scaling system:

SCALE3, $\mathrm{xr}=[\theta, \mathrm{nx}], \mathrm{yr}=[\theta, \mathrm{ny}], \mathrm{zr}=[\boldsymbol{\theta}, \mathrm{nz}], \mathrm{ax}=\mathrm{ax}, \mathrm{az}=\mathrm{az}$

; Contour the base:

CONTOUR, bzQ $(*, *, 0), /$ fill, nlevels $=25, /$ t3d, /noerase, zvalue $=0.0, /$ noclip,$\$$

$\mathrm{XSTYLE}=1, \mathrm{YSTYLE}=1, \mathrm{XRANGE}=[\theta, \mathrm{nx}], \mathrm{YRANGE}=[\theta, \mathrm{ny}], \mathrm{CHARSIZE}=4, \$$

XTITLE $={ }^{\prime} X$ ' , YTITLE $=$ ' $Y^{\prime}$, POS $=[0.1,0.1, n x, n y], \$$

$\mathrm{XTICKS}=4, \mathrm{XTICKNAME}=\mathrm{xt}, \$$

YTICKS $=4$, YTICKNAME $=y \mathrm{t}, \$$

$\mathrm{ZTICKS}=4, \mathrm{ZTICKNAME}=\mathrm{zt}$

; Set the 3D coordinate space with axes.

SURFACE, $[[\theta, \mathrm{nx}],[\theta, \mathrm{ny}]], / \mathrm{NODATA}, / \mathrm{SAVE}, /$ noerase $, / \mathrm{t} 3 \mathrm{~d}, \$$ 


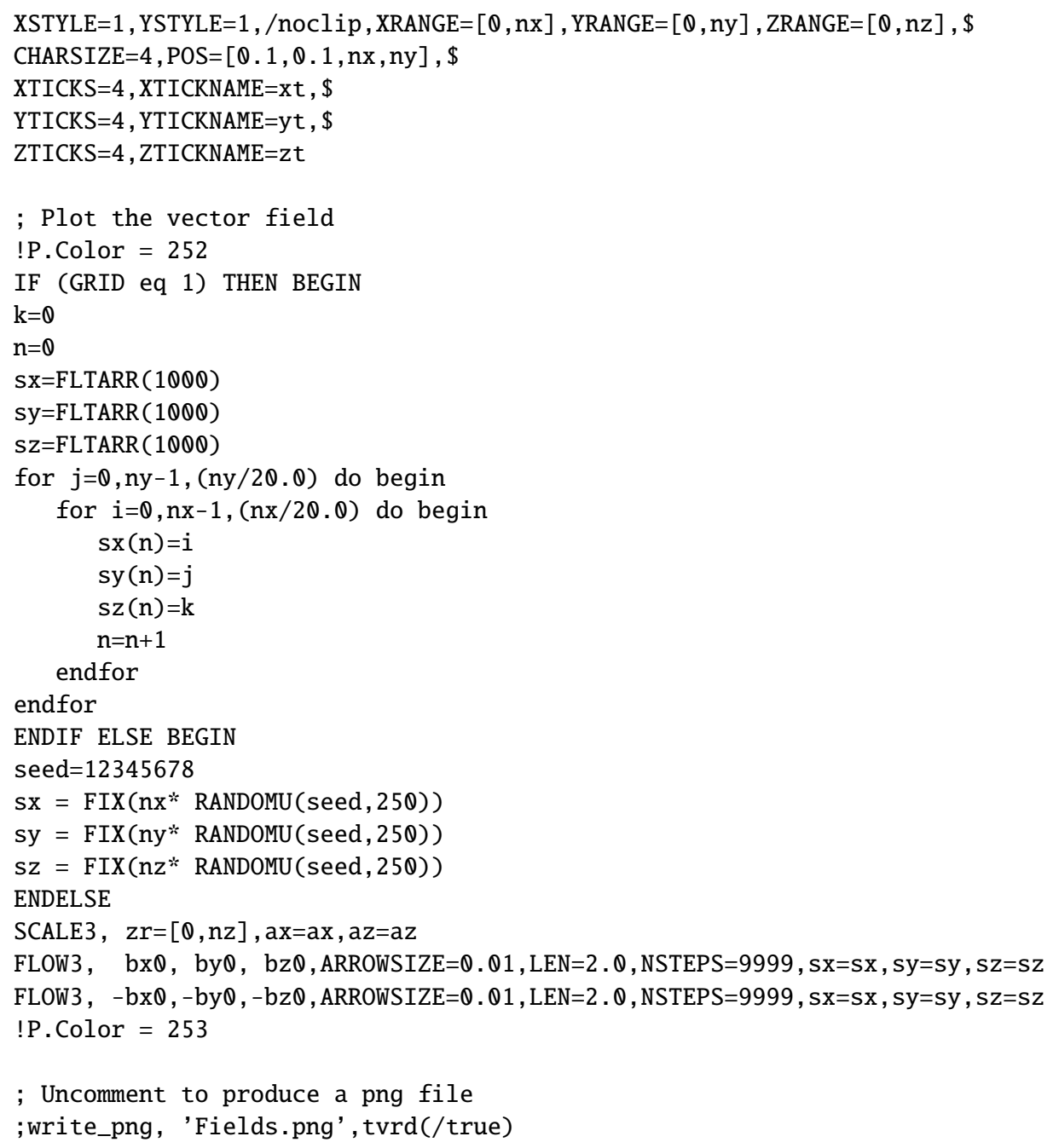

\section{Appendix D: Importing potential field data into Lare3d}

This appendix explains how the potential field data produced by BooTsik.f90 and stored in three files, bx.dat, by.dat, and bz.dat was imported into Lare $3 \mathrm{~d}$ and used as the initial magnetic field configuration. Firstly the data files bx.dat, by.dat, and bz.dat were copied into the same directory that Lare3d was run from. Changes were then made to four of the source files for Lare $3 \mathrm{~d}$.

The first source file to be edited was shared_data.F90, which can be found through the path src/core/shared_ data.F90. The following lines were added to the module shared_data to create allocatable arrays:

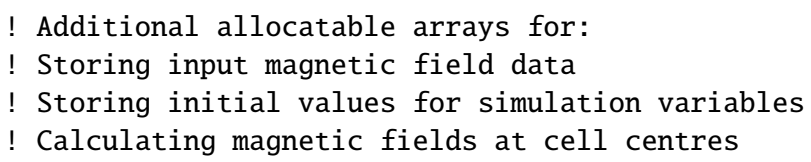

The second source file to be edited was control.f90, which can be found through the path src/control.f90. The changes that were made to this file are as follows. In the subroutine user_normalisation the normalisations were all set to 1.0. In the subroutine control_variables the number of gridpoints and the domain size were changed to match our input data, the runtime was set to 100.0 (this is measured in $\tau_{A}$ ), the resistivity was set to $5 \times 10^{-5}$, and the boundary conditions were set to user defined BC_USER. The following lines were then added to the end of the subroutine control_variables to read in the potential magnetic field data into allocatable arrays bx_init,by_init and bz_init:

! Here we allocate the arrays bx_init, by_init and bz_init

! We then read the files bx.dat, by.dat and bz.dat 
! and store the input magnetic fields into these arrays.

ALLOCATE ( bx_init ( $-2: \mathrm{nx}$ _global+2,-1:ny_global+2,-1:nz_global+2))

ALLOCATE ( by_init $\left(-1: n x_{-}\right.$global $+2,-2:$ ny_global $\left.+2,-1: n z \_g l o b a l+2\right)$ )

ALLOCATE( bz_init (-1:nx_global+2,-1:ny_global+2,-2:nz_global+2))

OPEN (unit=12, FORM = 'UNFORMATTED', STATUS='OLD', ACTION='READ', FILE = 'bx.dat')

READ (12) bx_init (-2:nx_global+2,-1:ny_global+2,-1:nz_global+2)

OPEN (unit=12, FORM = 'UNFORMATTED', STATUS='OLD', ACTION='READ', FILE = 'by.dat')

READ (12) by_init (-1:nx_global+2,-2:ny_global+2,-1:nz_global+2)

OPEN(unit=12, FORM = 'UNFORMATTED', STATUS='OLD', ACTION='READ', FILE = 'bz.dat')

READ (12) bz_init (-1:nx_global+2,-1:ny_global+2,-2:nz_global+2)

Finally in the subroutine set_output_dumps the time between snapshots was set to 10.0 (this is measured in $\tau_{A}$ ) and the dump masks 17-19 for the currents were set to . TRUE.

The third source file to be edited was initial_conditions.990, which can be found through the path src/initial_conditions.f90. The file initial_conditions.f90 sets the initial values of density $\rho$, energy $\varepsilon$, velocity field $\mathbf{v}$, and magnetic field $\mathbf{B}$ for each subdomain after domain decomposition. In this file we replace the subroutine set_initial_conditions with the following:

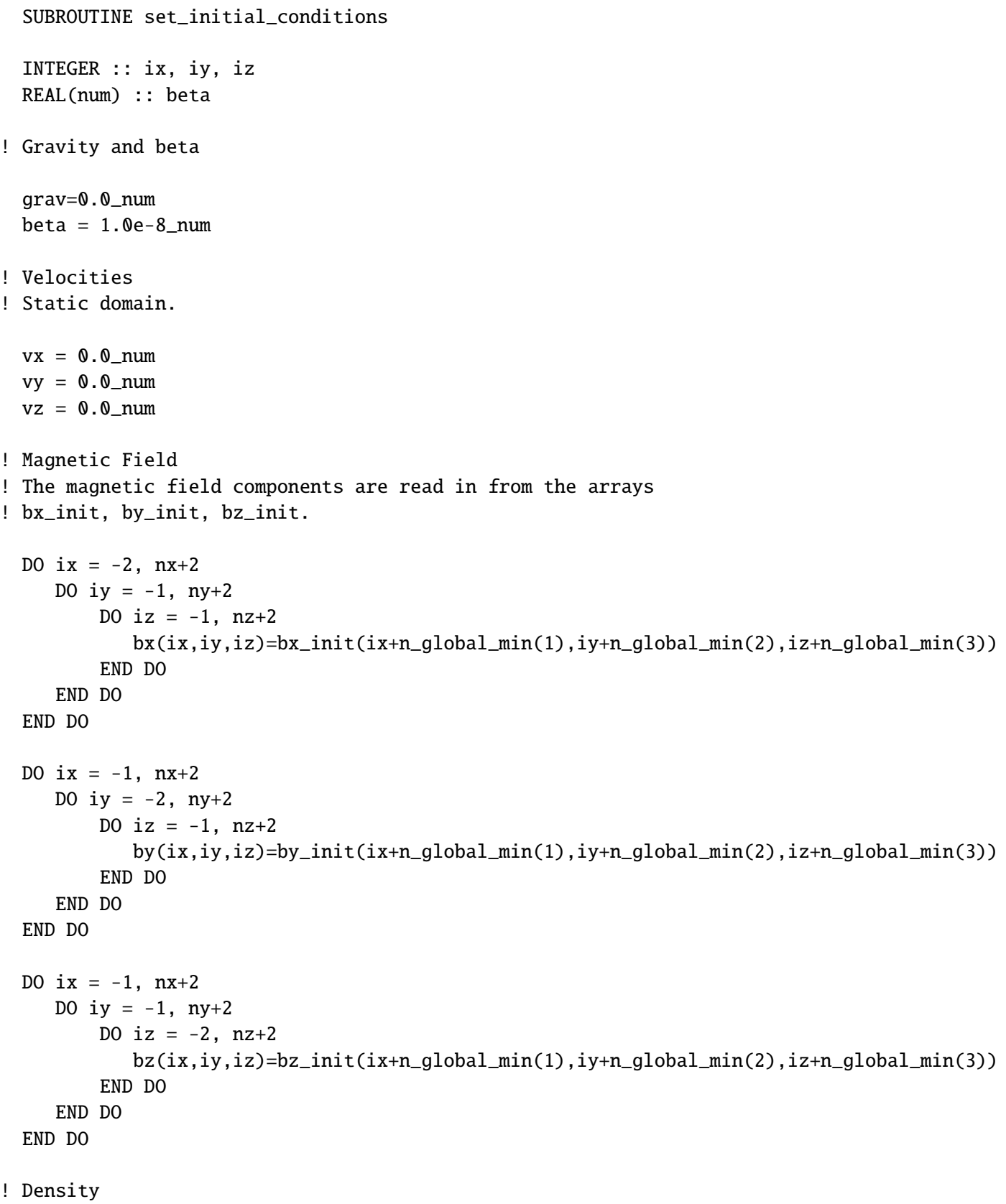


! In this case the density has been set equal to the

! square of the magnetic field strength.

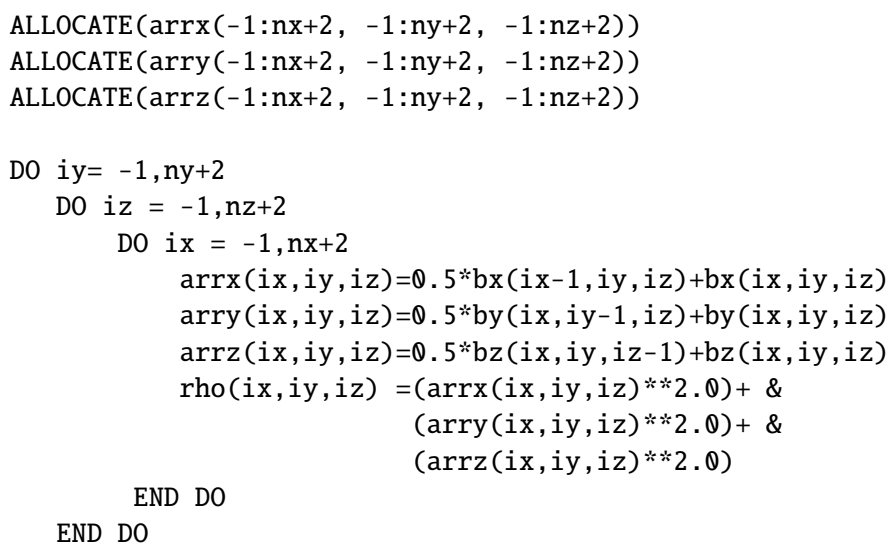

END SUBROUTINE set_initial_conditions

For each subdomain this subroutine sets gravity and the initial velocity field to zero. The subroutine sets the initial internal energy $\varepsilon$ such that the initial pressure $P$ is $5 \times 10^{-9}$ across the domain, imports the correct subdivision of the potential magnetic field, and sets the density equal to the square of the magnetic field strength $\rho=B^{2}$. Finally these initial values are stored in arrays rho $\theta$, bx $\theta$, by $\theta$, bz $\theta$, energy $\theta$.

The fourth and final source file to be edited was boundary.f90, which can be found through the path src/initial_boundary.f90. This boundary conditions in this file were edited so that at every boundary the density $\rho$, internal energy $\varepsilon$, and magnetic field components $\mathrm{bx}, \mathrm{by}, \mathrm{bz}$ were all set to their initial values rho $\mathbb{\theta}, \mathrm{bx} \boldsymbol{\theta}, \mathrm{by} \boldsymbol{\theta}, \mathrm{bz} \boldsymbol{\theta}$, energy $\mathbb{\theta}$. An example of this is given below, the example given is for the magnetic fields at minimum $x$-boundary:

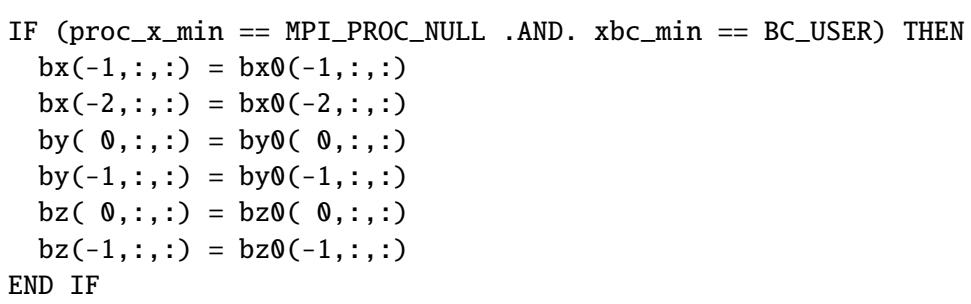

Finally the velocities at all boundaries at both full and half timesteps were all set to zero. An example of this is given below, the example given is for the full timestep velocities at minimum $x$-boundary:

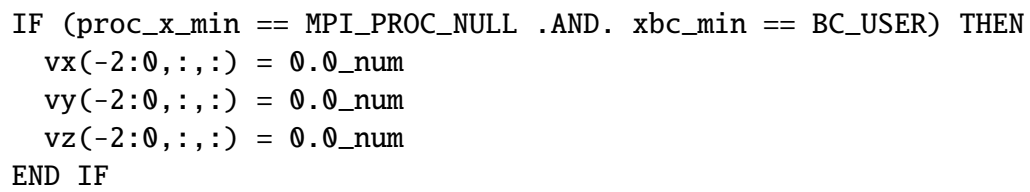

